\title{
Mood and Comparative Judgment: Does Mood Influence Everything and Finally Nothing?
}

\author{
CHENG QIU \\ CATHERINE W. M. YEUNG*
}

\begin{abstract}
Research indicates that mood can influence evaluation of a product when considered in isolation. However, little is known about its influence on comparisons among several alternatives. Four experiments assessed the nature of this influence. When evaluating each option individually upon encountering it, happy participants reported greater preferences for the first encountered option than unhappy participants. When withholding evaluations until having seen all options, however, happy participants reported greater preferences for the last encountered option than unhappy participants. Which comparison strategy was employed, and consequently the impact of mood on preferences, depended on the similarity of choice alternatives in terms of appearance versus descriptive features.
\end{abstract}

$\mathrm{T}$ he affect that consumers happen to be experiencing at the time they evaluate a stimulus can provide information about their liking for the stimulus and, therefore, can influence their evaluation of it. Research in both social psychology and consumer judgment has demonstrated this influence and has circumscribed the conditions in which the effect occurs (Pham 1998; Pham and Avnet 2004; Pham et al. 2001; Schwarz and Clore 1996; Yeung and Wyer 2004). With few exceptions (e.g., Shiv and Fedorikhin 1999, 2002), most previous research has focused on the influence of affect when a stimulus is considered individually. Our understanding of the role of affect in consumer judgment is therefore restricted to situations where products are evaluated in isolation. The challenge of extending this knowledge to the choice context lies in the fact that choices are largely based on differences between alternatives rather than the indepen-

\footnotetext{
*Cheng Qiu is assistant professor of marketing at the Faculty of Business and Economics, University of Hong Kong, Pokfulam Road, Hong Kong (cqiu@business.hku.hk). Catherine Yeung is assistant professor of marketing at the NUS Business School, National University of Singapore, Singapore 117592, Republic of Singapore (cyeung@nus.edu.sg). Corresponding author: Catherine Yeung. The two authors contributed equally to this work, and their names are arranged in alphabetical order. This research was conducted during the first author's PhD study at the National University of Singapore. The authors thank Bob Wyer, Leong Siew Meng, Lee Yih Hwai, and the JCR editor, associate editor, and reviewers for their insightful comments and suggestions. In addition, the authors thank Colin Soh, Mak $\mathrm{Ka}$ Weng, and Sun $\mathrm{Li}$ for assistance in data collection. The support of grant R-316-000-052-112 from the National University of Singapore and the support of the NUS Marketing Department subject pool are gratefully acknowledged.
}

John Deighton served as editor and Mary Frances Luce served as associate editor for this article.

Electronically published September 21, 2007 dent evaluations of the alternatives. Thus, if an affect influences evaluations of all the alternatives in a similar way and to a similar extent, it may not end up having any impact on their choice probabilities (see Huber [2004] for a similar comment).

In the present research, we show that the mood people happen to be experiencing can have different effects on their preferences for choice alternatives, depending on the order in which the alternatives are considered. Specifically, we suggest that consumers are likely to incorporate the mood they are experiencing into the first evaluation they construct in the course of making a comparison. Once this is done, however, their mood is less likely to influence the evaluations that are constructed subsequently. One implication of this proposition is that the influence of mood on preferences depends on the time at which the first evaluation is made. If consumers who are exposed to multiple options evaluate each option spontaneously at the time they first encounter it, their mood may influence their evaluation of the first presented option. However, if consumers withhold their evaluation until they have seen all the options available, they may evaluate the last presented (most recent) option first, and their mood may influence their evaluation of this option.

In the sections that follow, we first review the literature on the influence of affect on independent judgment and discuss its theoretical implications for comparative judgment. We then report four experiments that examine these implications.

\section{AFFECT, INDEPENDENT JUDGMENT, AND COMPARATIVE JUDGMENT}

Individuals who are called upon to evaluate an object often use the affect they are experiencing as information 
about how much they like it (Adaval 2001; Pham 1998; Pham et al. 2001; Schwarz 1990; Schwarz and Clore 1983). In some cases, the affect they experience may actually be evoked by the stimulus being judged (Schwarz 2001; Shiv and Fedorikhin 1999, 2002; Yeung and Wyer 2005, experiment 1). However, individuals usually cannot distinguish clearly between the different sources of affect that they experience at any given time. Consequently, if they happen to be in a good mood or bad mood when they evaluate a target, they may (mis)attribute this feeling to the target and use it as a basis for the evaluation (Schwarz and Clore [1983, 1988]; see Pham [2004] for a discussion of the logic behind people's reliance on feelings in judgment).

For one's feelings to influence the evaluation of a target, these feelings must be perceived as genuine responses toward the target (Strack 1992). One's feelings may have little impact on the evaluation of a target if the type of target being evaluated does not normally elicit affective reactions (Yeung and Wyer 2004), if one has concerns about the relevance of feelings as a basis for the evaluation (Pham 1998), or if one's attention is called to other factors that could potentially account for the feelings (Schwarz and Clore 1983). In a classic study by Schwarz and Clore (1983), for example, participants reported their life satisfaction to be higher on sunny days than on rainy days, indicating that the feelings elicited by the weather were misattributed to their life as a whole. However, the effect of negative feelings was eliminated when the experimenter happened to mention the weather, thus directing participants' attention to this source of their negative feelings. While the influence of positive mood is less clear in this study, this finding implies that when people attribute their negative feelings to one target, the informational value of these feelings for evaluating another target is often discounted, and hence the impact of these feelings on the evaluation of the latter target is minimal. This attribution effect has been found applicable to positive mood in a product evaluation context (Gorn, Goldberg, and Basu 1993) and has been conceptually replicated in other research (Novemsky et al. 2007; Schwarz, Servay, and Kumpf 1985; Siemer and Reisenzein 1998).

\section{Affect Attribution and Comparative Judgment}

The attributional effect discussed above has implications for the conditions of concern in the present research. Suppose consumers who are confronted with a choice among multiple options evaluate each option separately and then compare these evaluations to arrive at a preference. They are likely to attribute their feelings to the first option they evaluate, as if they are evaluating this option individually. Thus, they will evaluate it more favorably when they are in a good mood than when they are in a bad mood. Having made this evaluation, however, they may discount the informational value of their feelings to the options they consider subsequently. Mood will therefore have a minimal effect on the evaluations of these latter options. When they later compare the evaluations to arrive at a preference, their preference for the first evaluated option will increase with the mood that they are experiencing.

To nail down the specific influence of mood on comparative judgment, we have to specify which of the options in a choice set is the first one to be evaluated. In some cases, consumers may evaluate the options in the order in which they are presented. As such, the first option they evaluate is likely to be the first presented option. Mood will therefore influence the evaluation of this particular option (rather than the evaluations of the subsequently presented options) and hence the preference for this option. In other cases, consumers may forgo making an evaluation of any option until they have seen all of the options available. Under this situation, the first option they evaluate is likely to be the last one they have encountered, which is most salient to them at the time of evaluation. Their mood should therefore influence their evaluation of the last option rather than the preceding ones. Consequently, they should prefer the last presented option more if they are in a good mood than if they are in a bad mood.

It is of course conceivable that mood exerts its influence at the time consumers view each option rather than at the time they evaluate a particular option. If this is so, mood may influence evaluations of all the options regardless of the considerations we discussed above. Although no research bears directly on this possibility, a study by Srull (1987) suggests that this is unlikely to be the case. In some conditions of Srull's study, participants received product information and evaluated the product right after their mood had been induced. In other conditions, they received the information while they were in a good or bad mood but did not evaluate the product until after their mood had dissipated. Mood influenced their evaluations in the first condition but not in the second. This suggests that mood influences an evaluation only if it coincides with the timing of evaluation. Putting it another way, the finding implies that mood enters an evaluation at the precise time when the evaluation is actually constructed. In the present context, this suggests that although participants may be experiencing a positive or negative mood at the time they view a set of options, this mood may not influence the evaluations of the options if these evaluations are not constructed at that point of time.

The above considerations lead to the following hypothesis:

H1: When consumers compare among multiple options, their mood will influence their preference for the first option they evaluate. More specifically, consumers will evaluate this option more positively, and will be more likely to choose it, if they are in a positive mood than if they are in a negative mood. In contrast, their mood will have a minimal effect on evaluations of the options that are evaluated subsequently. 
FIGURE 1

\section{STIMULI EMPLOYED IN EXPERIMENT 1}
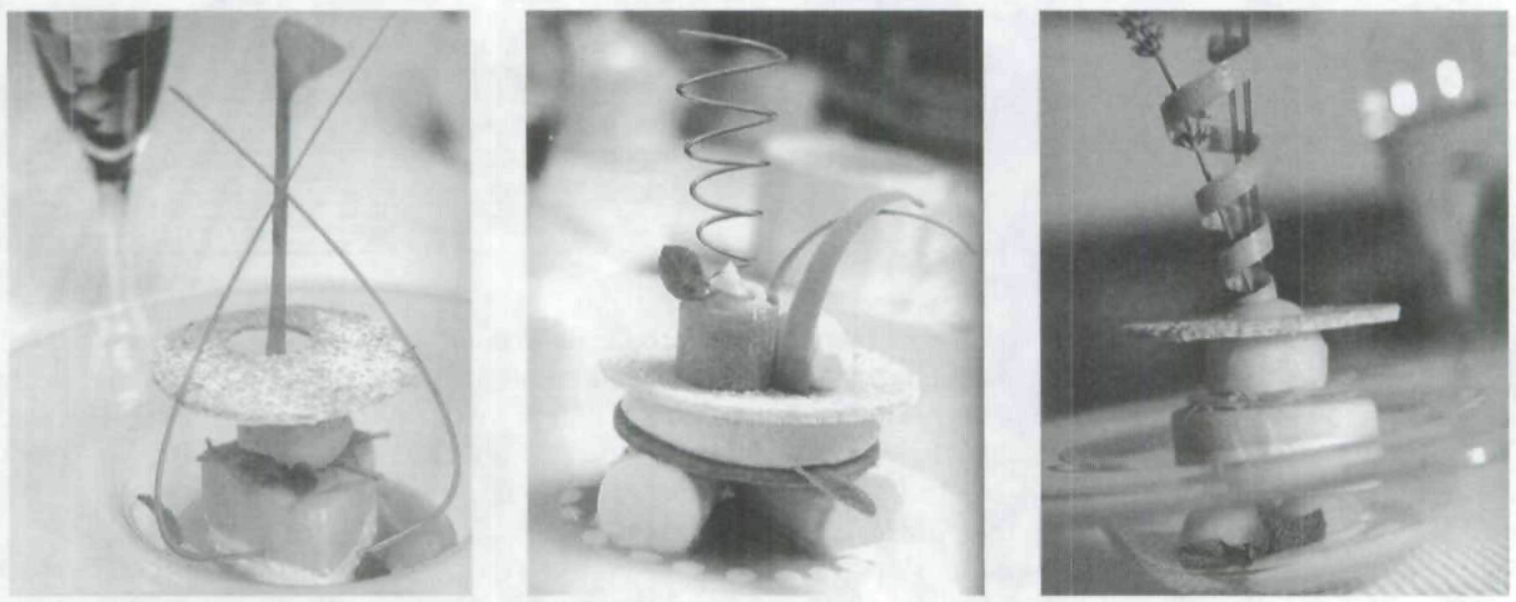

Source.-Sweet Seasons: Fabulous Restaurant Desserts Made Simple, by Richard Leach; photography by Boyd Hagen (New York: Wiley \& Sons, 2001), 70, 72 , and 210 .

\section{Situational Determinants of Comparison Strategy}

The point at which consumers evaluate choice alternatives in the course of comparison may be determined by several factors. Most obviously, participants might be explicitly told either to evaluate each option individually at the time they encounter it or to wait until they have seen all of the available options before making an evaluation. However, characteristics of the options themselves could also influence the strategy that participants employ. For example, suppose the options are similar in quality and function but differ only in appearance. In this case, holistic, appearance-based evaluations will be perceived as a diagnostic basis for preference formation. To this extent, a schematic assessment of the first presented option would be triggered upon sight, which would then lead to an affect-based evaluation of the option (Pham et al. 2001; Yeung and Wyer 2004). However, suppose consumers expect the options to differ in terms of descriptive features that are central to their choice. Then, they may be more inclined to defer their evaluation until the information about the last option has been reviewed. This prediction is generally consistent with the literature on comparative judgment, which suggests that individuals who expect to make preference judgments may not evaluate an initial object in terms of its own descriptive features prior to seeing a comparison object. Rather, they are likely to wait until the features of the comparison object are also known in order to evaluate the features in a relative sense. Moreover, to the extent that they defer their judgment, they are likely to begin by considering the most recently encountered option-the last option-as it is more salient and accessible from memory than the preceding option(s) (see Houston, Sherman, and Baker [1989] for a discussion of a similar process; also see Wang and Wyer [2002]).

The above possibilities were examined in the research to be reported. In experiment 1 , the options that we employed had the same descriptive characteristic but different external appearance. We assumed that participants would form an evaluation of the first presented option at the time they encountered it. Their mood would therefore influence this evaluation but not the evaluations of options that were presented subsequently. Thus, we expected participants to have a stronger preference for the first option if they were in a good mood than if they were in a bad mood. In the second experiment, we asked participants to consider options that differed not only in external appearance but also in a descriptive characteristic. In this case, we expected participants to defer evaluating the options until all the options had been presented. Their mood would therefore influence their evaluations of the last presented option rather than those of the preceding options. Hence, they would report a stronger preference for the last option when they were in a good mood than when they were in a bad mood. After reporting the results of these two experiments, we will present two other experiments that confirmed our assumptions about the processes that underlie the findings.

\section{EXPERIMENT 1}

\section{Method}

Stimulus Materials. We selected bakery products as target stimuli. Evaluations of bakery products are typically based on two criteria-flavor (a descriptive characteristic) and presentation (external appearance). We chose three options with the same flavor (mango) but different presentations. The photographs of these options (see fig. 1) were printed on high-quality photo paper. All photographs had a similar, yellowish tone to control for the possible influence of color on the feelings they elicited. 
TABLE 1

CHOICES AND INDEPENDENT EVALUATIONS AS A FUNCTION OF MOOD-EXPERIMENT 1

\begin{tabular}{|c|c|c|c|c|c|c|}
\hline & \multicolumn{3}{|c|}{ Choice share (\%) } & \multicolumn{3}{|c|}{ Independent evaluations } \\
\hline & $\begin{array}{c}\text { 1st } \\
\text { option }\end{array}$ & $\begin{array}{l}\text { 2nd } \\
\text { option }\end{array}$ & $\begin{array}{c}\text { 3rd } \\
\text { option }\end{array}$ & $\begin{array}{c}\text { 1st } \\
\text { option }\end{array}$ & $\begin{array}{l}\text { 2nd } \\
\text { option }\end{array}$ & $\begin{array}{c}\text { 3rd } \\
\text { option }\end{array}$ \\
\hline Positive mood $(n=65)$ & 49.2 & 26.2 & 24.6 & 4.32 & 3.90 & 3.89 \\
\hline Negative mood $(n=66)$ & 25.8 & 39.4 & 34.8 & 3.55 & 4.27 & 4.30 \\
\hline
\end{tabular}

To ensure that these options were comparable in favorableness, 62 participants who did not participate in the main study were each shown one of the options and indicated both how much they liked it $(0=$ not at all; $10=$ very much) and how they felt about it $(-5=$ very bad; $+5=$ very good). All options were equally favorable $(7.84,7.56$, and 7.64 for the first, second, and third options shown in fig. 1 , respectively; $F<1)$ and elicited similar feelings (3.46, 3.55 , and 3.71 , respectively; $F<1$ ).

Design and Procedure. Participants were 131 students at the National University of Singapore who participated in exchange for course credit. They were randomly assigned to the two induced mood (positive vs. negative) conditions. They performed two ostensibly unrelated tasks-one induced their mood, and the other concerned product choice. In the first task, participants were induced to experience either a positive or a negative feeling with a procedure similar to that employed by Schwarz and Clore (1983). Specifically, they were told that the study concerned the construction of a database on the personal experiences of college students. Participants in the positive (negative) mood condition were asked to identify a recent event that was very important to them and that made them feel happy (unhappy) whenever they thought about it. They were asked to imagine the experience in as much detail as possible, to try to reexperience the feelings they had at the time, and then to write down a description of those feelings and the events that elicited them. They spent approximately 20 minutes writing their descriptions.

After completing the mood induction task, participants were introduced to the second task with instructions that we were conducting a series of surveys that required participants to choose among different products of the sort they might encounter in daily life and that the particular survey they would complete was concerned with choices among bakery products. On this pretext, we informed participants that they would see photographs of three products that had the same flavor. The photographs were presented in separate folders denoted A, B, and C. In each folder, the description "mango flavor" was printed below the photograph. Participants were first instructed to open folder A, look at the photograph for 5 seconds, close the folder, and then to repeat the procedure for the B and C folders, respectively. The sequence in which these folders were labeled and shown was counterbalanced.
The experimenter then passed out the choice questionnaire, and participants indicated their choices. They then evaluated each option individually on the next page. For each option, they indicated how much they like it $(0=$ not at all; $10=$ very much) and how good it is $(-5=$ very bad; $+5=$ very good).

To confirm the effectiveness of the mood induction procedure, participants were asked to report their feelings after having described the personal experience. These feelings were assessed by four scales pertaining to how happy, good, unhappy, and bad they were feeling; all ranged from zero (not at all) to 10 (very much). The average of each participant's responses to the second two items was subtracted from the average of his or her responses to the first two items to provide an overall index of the positive feeling that the participant was experiencing.

\section{Results}

Mood Manipulation Check. The mood induction procedure was successful. Participants reported feeling happier after describing their experience in the positive mood condition than in the negative mood condition ( 4.95 vs. -3.14 ; $\left.F(1,129)=172.52, p<.001, \eta_{p}^{2}=.57\right)$.

Choices and Independent Evaluations. Choice shares of the three options as a function of the two mood conditions are reported in the left half of table 1 . An overall 2 (mood) $\times 3$ (option) chi-square analysis of these proportions revealed an association between participants' mood and their choices $\left(\chi^{2}(2)=7.73, p<.05\right)$. To understand whether this association was specifically due to an impact of mood on choice of the first option, two additional chi-square tests were conducted (Agresti 1996). First, a chi-square test based on the last two columns of the $2 \times 3$ contingency table did not indicate any association between mood and choices of the last two options $\left(\chi^{2}(1)=.02, p>.50\right)$. Choices of the last two options were therefore combined and compared with choice of the first option. The result showed that happy and unhappy participants differed in their choice of the first option relative to their choices of the other two options combined $\left(\chi^{2}(1)=7.71, p<.01\right)$. As predicted, the choice share of the first option was higher when participants were happy $(49.2 \%)$ than when they were unhappy $(25.8 \% ; z=2.79$, $p<.05)$.

In principle, the shift in choice shares across the two mood 
conditions may be driven by changes in the attractiveness of the first option or by opposite changes in the attractiveness of the other options. However, if participants had indeed attributed their mood to the first option, as we predicted, the shift in choice shares should be driven by changes in the attractiveness of the first option in particular. We next evaluate this possibility. Recall that participants evaluated each of the three options by indicating the extent to which they liked the option and how good the option was. Their responses to these items for each option were averaged to form an index of how much they liked the option. These evaluations are summarized in the right half of table 1. A 2 (mood) $\times 3$ (evaluations of the three options) mixed ANOVA revealed a significant interaction between mood and the three evaluations $(F(2,258)=4.48, p=.01$, $\left.\eta_{p}^{2}=.03\right)$. Planned comparisons revealed that happy participants evaluated the first option more favorably than unhappy participants (4.32 vs. 3.55 , respectively; $t(129)=$ $\left.1.97, p=.05, \eta_{p}^{2}=.03\right) .{ }^{1}$ However, the two groups did not differ appreciably in their evaluations of the second option ( 3.90 vs. $4.27 ; t(129)=1.18, p=.24)$ and the third option $(3.89$ vs. $4.30 ; t(129)=1.25, p=.21)$.

\section{Discussion}

In this experiment, we show that preferences for the first presented option increased with the mood that participants were in and that this was due largely to the effect of mood on the evaluations of the first presented option, rather than the evaluations of the subsequently presented options.

To ensure that our results were not dependent on the number of options that participants considered, we performed a supplementary study that was identical to the main experiment but involved only two options (specifically, the first two options shown in fig. 1). Fifty-five participants were randomly assigned to the two mood conditions. The conclusions drawn from this study are identical to those that emerge from the main experiment. That is, $69.0 \%$ of happy participants chose the first option, whereas only $38.5 \%$ of unhappy participants did so $\left(\chi^{2}(1)=5.15, p<.05\right)$. Moreover, planned comparisons of the evaluations of each option indicated that happy participants evaluated the first option more favorably than unhappy participants did (4.93 vs. 3.77 , respectively; $t(53)=2.33, p<.05, \eta_{p}^{2}=.09$ ), whereas their evaluations of the second option did not differ (4.34 vs. 4.23 , respectively; $t<1$ ). Although a mixed ANOVA showed that the interaction of mood and evaluations was not significant $(F(1,53)=1.76, p=.19)$, the planned comparisons were consistent with our predictions.

Both experiment 1 and the 2-option replication showed an influence of mood on the preference for the first presented option. According to hypothesis 1 , however, this should not be the case if the first presented option is not the first one

'Here and subsequently, planned comparisons are $t$-tests conducted using the error term of the full ANOVA model. See Keppel (1991, 122-23) for further discussion. that participants evaluate. Experiment 2 examined this possibility.

\section{EXPERIMENT 2}

This experiment was similar to experiment 1 except that the choice alternatives differed in flavor as well as appearance. Participants were told at the outset that the alternatives had different flavors and that they would learn each option's flavor only when they saw its photograph. Therefore, they would be in anticipation of additional, diagnostic information until they had received information of the last option. This was expected to increase the likelihood that participants would withhold making any evaluations until they had seen all of the options. We therefore predicted that participants' mood would influence their evaluation of the last presented option rather than the preceding ones. Consequently, they would prefer the last option to a greater extent when they were in a good mood than when they were in a bad mood.

\section{Method}

Stimulus Materials. Three pies (blueberry, almond, and plum) were selected as stimulus materials. Photographs of these options, shown in figure 2 , were evaluated by 63 participants who did not take part in the main experiment. The three options were rated similarly both in favorableness (7.51, 7.39, and 7.59 for the first, second, and third options shown in the figure, respectively; $F<1$ ) and in the feelings they elicited $(4.01,3.94$, and 3.89 , respectively; $F<1)$.

Design and Procedure. One hundred students at the National University of Singapore participated in the experiment in exchange for course credit. They were assigned randomly to the two induced mood conditions (positive vs. negative). As in the first experiment, participants first engaged in a mood induction task, followed by a choice task. When they were introduced to the latter task, they were told that the bakery products they would be considering had different flavors and that they would know each option's flavor only when they saw its photograph from a folder. Each folder showed a photograph of a bakery product with a label below the photograph describing its flavor. After exposure to the three products, participants indicated choices and made ratings as they did in experiment 1 .

\section{Results}

Mood Manipulation Check. The mood induction procedure was successful. Participants reported being happier after describing their experience in the positive mood condition than in the negative mood condition (5.52 vs. -3.11 ; $\left.F(1,98)=192.76, p<.001, \eta_{p}^{2}=.66\right)$.

Choices and Independent Evaluations. In contrast to experiment 1 , the effects of mood on choice were expected to localize in the last presented option. The findings were shown in the left half of table 2. Although the overall chi- 
FIGURE 2

\section{STIMULI EMPLOYED IN EXPERIMENT 2}
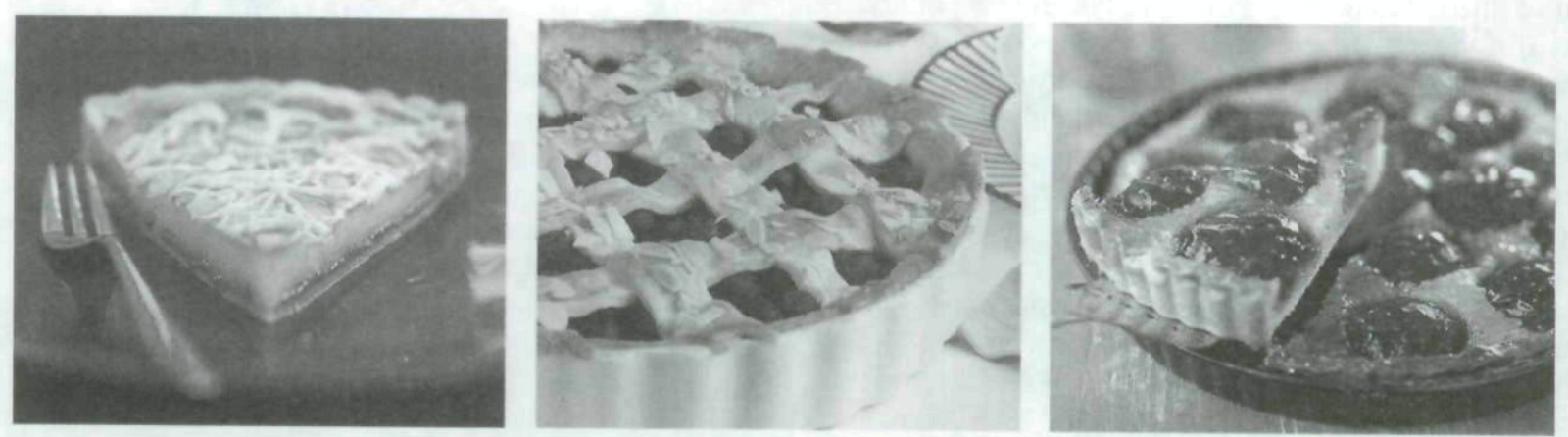

SOURCE.-Million Menus (London: Octopus Publishing Group, 2004), 242, 244, and 248.

square test based on the $2 \times 3$ contingency table was only marginally significant $\left(\chi^{2}(2)=5.35, p=.07\right)$, two other tests support our prediction that mood would influence the preference for the last option. First, the effect of mood on choices of the first two options did not differ across the two mood conditions $\left(\chi^{2}(1)=.17, p>.50\right)$. Therefore, choices of the first two options were combined and compared with the choice of the last option. The result showed that happy and unhappy participants differed in their choice of the last option relative to their choices of the preceding options $\left(\chi^{2}(1)=5.19, p<.05\right)$. More specifically, participants were more likely to choose the last option when they were happy $(48 \%)$ than when they were unhappy $(26 \% ; z=2.27, p<$ $.05)$.

Independent evaluations of the alternatives, shown in the right half of table 2, revealed an analogous pattern. A mixed ANOVA of the three evaluations as a function of mood yielded a marginally significant interaction of mood and the three evaluations $\left(F(2,196)=2.53, p=.08, \eta_{p}^{2}=.03\right)$. Planned comparisons indicated that participants liked the last option more when they were in a positive $\operatorname{mood}(M=4.39)$ than when they were in a negative $\operatorname{mood}(M=3.34$; $\left.t(98)=2.31, p<.05, \eta_{p}^{2}=.05\right)$. However, mood influenced the evaluation of neither the first option (3.82 vs. $4.12 ; t<1)$ nor the second ( 3.56 vs. $3.71 ; t<1)$.

\section{Discussion}

In this experiment, we showed that preferences for the last presented option increased with the mood that partici- pants were in and that this was due largely to the effect of mood on the evaluation of the last presented option rather than the evaluation of the first presented option. These results clearly differ from those obtained in the first experiment. We have attributed this difference to the different time at which evaluations were made in the two experiments. That is, participants who knew that the options differed only in appearance might have evaluated each option at the time it was presented, as in experiment 1 . However, when they expected the options to differ with respect to attributes that are more central to their evaluation, they might have deferred their evaluation until all of the options had been presented. However, a question concerning the validity of this interpretation may arise from the fact that the time at which participants made their evaluations was neither directly assessed nor manipulated. Experiment 3 addressed this ambiguity.

\section{EXPERIMENT 3}

The purpose of this experiment was to validate the assumptions that we made in the previous experiments concerning the time at which the first evaluation was constructed and hence to provide evidence of the underlying process that we proposed. To do so, we included process baseline conditions in which participants were either instructed to evaluate each option as it was presented or not to make any evaluations until they had seen all the options (see Pham [1998], experiment 2, for a similar design). Results in these

TABLE 2

CHOICES AND INDEPENDENT EVALUATIONS AS A FUNCTION OF MOOD-EXPERIMENT 2

\begin{tabular}{|c|c|c|c|c|c|c|}
\hline & \multicolumn{3}{|c|}{ Choice share (\%) } & \multicolumn{3}{|c|}{ Independent evaluations } \\
\hline & $\begin{array}{c}\text { 1st } \\
\text { option }\end{array}$ & $\begin{array}{l}\text { 2nd } \\
\text { option }\end{array}$ & $\begin{array}{c}\text { 3rd } \\
\text { option }\end{array}$ & $\begin{array}{c}\text { 1st } \\
\text { option }\end{array}$ & $\begin{array}{c}\text { 2nd } \\
\text { option }\end{array}$ & $\begin{array}{c}\text { 3rd } \\
\text { option }\end{array}$ \\
\hline Positive mood $(n=50)$ & 24 & 28 & 48 & 3.82 & 3.56 & 4.39 \\
\hline Negative mood $(n=50)$ & 38 & 36 & 26 & 4.12 & 3.71 & 3.34 \\
\hline
\end{tabular}


TABLE 3

TIMING OF THE CONSTRUCTION OF THE FIRST EVALUATION-EXPERIMENT 3

\begin{tabular}{|c|c|c|c|}
\hline & No instruction & $\begin{array}{l}\text { Immediate } \\
\text { judgment }\end{array}$ & $\begin{array}{l}\text { Deferred } \\
\text { judgment }\end{array}$ \\
\hline Same flavor & $5.16(n=41)$ & $4.63(n=34)$ & $4.19(n=32)$ \\
\hline Different flavor & $4.43(n=40)$ & $4.91(n=32)$ & $4.41(n=32)$ \\
\hline
\end{tabular}

conditions were then compared with those obtained under conditions similar to those examined in previous experiments, where participants did not receive any instructions about how to process the information. If participants from a baseline condition reach similar decisions as the corresponding uninstructed participants, the process imposed on the baseline participants provides at least a paramorphic, and possibly isomorphic, representation of the process followed by the uninstructed participants. However, if the two groups of participants reach different decisions, it is unlikely that the process followed by the uninstructed participants resembles the one specified in the baseline condition.

\section{Method}

Design and Stimulus Materials. Participants were 211 students at the National University of Singapore who participated in exchange for course credit. They were randomly assigned to the 12 conditions of a 2 (induced mood: positive vs. negative) $\times 2$ (flavor: same flavor vs. different flavor) $\times 3$ (process type: no instruction vs. immediate judgment vs. deferred judgment) factorial design. Participants were asked to consider two options, which were described as having either the same flavor or different flavors. In both cases, we used the first two photographs in figure 1 to represent the options. The two options were described as having a mango flavor in same-flavor conditions and were described as having a peach flavor and a mango flavor (pretested to be equally favorable), respectively, in different-flavor conditions.

Procedure. All participants went through a mood induction procedure similar to that employed in the previous experiments and then moved on to a comparison task that was ostensibly unrelated to the mood induction task. In immediate-judgment conditions, participants evaluated each option immediately after seeing it. Therefore, the first option being evaluated, by the procedure administered, was the first option participants encountered. Evaluations were reported along scales pertaining to how much they liked it and also how good it was; each ranged from zero (not at all) to 10 (very). Then, after both options were presented and evaluated, they reported their preferences for the two options on a relative preference scale ranging from one (definitely the first option) to 12 (definitely the second option).

Participants in deferred-judgment conditions were told "because there are two different options, try not to make any judgments until you have seen both. That is, . . . try not to make any judgments until after you have seen the second one." We expected that the first option participants would evaluate would be the second option they encountered. After seeing both options, participants first reported their preferences and then evaluated the individual options.

Participants in no-instruction conditions, like those in the first two experiments, were not given any instruction on when they should evaluate the options. These participants first saw the two options, reported their preferences, and followed with their evaluations of the options separately.

Finally, to confirm our assumptions about the time at which participants made their evaluations, participants reported their agreement with each of the following statements: (a) I had formed an evaluation of the first dessert before I saw information on the second dessert, and $(b)$ I did not start to form any evaluation of either dessert until after I had seen information on both of them. Both ratings were made along a scale from one (strongly disagree) to seven (strongly agree). Each participant's response to the second item was reverse coded and averaged with his/her response to the first item to form a single index of the disposition to evaluate the first option immediately after seeing it.

\section{Results}

Mood Manipulation Check. The mood induction procedure was successful. Participants reported feeling happier after describing their experience in the positive mood condition than in the negative mood condition $(6.10 \mathrm{vs} .-3.78$; $\left.F(1,199)=803.32, p<.001, \eta_{p}^{2}=.80\right)$.

Timing of the First Evaluation. Data pertaining to the timing of constructing the first evaluation are summarized in table 3. (Numbers are overall index, with larger numbers indicating a greater disposition to evaluate the first option immediately after seeing it.) An ANOVA of the data as a function of process type, flavor type, and mood yielded an interaction of process type and flavor type $(F(2,199)=$ $3.69, p<.05, \eta_{p}^{2}=.04$ ) that was independent of mood (the three-way interaction was not significant; $F(2,199)=$ $1.47, p=.23)$. As expected, participants in no-instruction conditions were more inclined to evaluate the first option immediately when the alternatives had the same flavor 
TABLE 4

RELATIVE PREFERENCE AS A FUNCTION OF MOOD, PROCESS TYPE, AND FLAVOR CONDITIONS-EXPERIMENT 3

\begin{tabular}{lccc}
\hline \hline & No instruction & $\begin{array}{c}\text { Immediate } \\
\text { judgment }\end{array}$ & $\begin{array}{c}\text { Deferred } \\
\text { judgment }\end{array}$ \\
\hline Same flavor: & & & \\
Positive mood & $4.43(n=21)$ & $5.06(n=18)$ & $6.50(n=16)$ \\
Negative mood & $6.40(n=20)$ & $6.69(n=16)$ & $5.06(n=16)$ \\
Difference & -1.97 & -1.63 & 1.44 \\
$\begin{array}{l}\text { Different flavor: } \\
\text { Positive mood }\end{array}$ & $6.62(n=21)$ & $4.94(n=16)$ & $5.56(n=16)$ \\
Negative mood & $4.42(n=19)$ & $7.13(n=16)$ & $4.56(n=16)$ \\
Difference & 2.20 & -2.19 & 1.00 \\
\hline $\begin{array}{l}\text { Note.-Pelative preference was measured on a } 12 \text {-point scale }(1=\text { strongly prefer the first } \\
\text { option; } 12=\text { strongly prefer the second option). }\end{array}$ &
\end{tabular}

$(M=5.16)$ than when they had different flavors $(M=$ $\left.4.43 ; t(199)=2.63, p<.01, \eta_{p}^{2}=.03\right)$. However, when participants received instruction of when they should evaluate the options, the timing of the first evaluation did not differ across the two flavor-type conditions $(t<1$ in both cases). Participants reported a stronger tendency to evaluate the first option immediately in immediate-judgment conditions $(M=4.78)$ than in deferred-judgment conditions $\left(M=4.30 ; t(199)=2.08, p<.05, \eta_{p}^{2}=.02\right)$.

Relative Preference. Data on relative preference are summarized in table 4. An ANOVA showed a significant three-way interaction of mood, flavor type, and process type $\left(F(2,199)=5.19, p<.01, \eta_{p}^{2}=.05\right)$. Our predictions were evaluated in a series of planned comparisons and interaction contrasts using the error term from the full ANOVA model (Keppel 1991).

First, to confirm the conclusions drawn from our earlier studies, we considered the no-instruction conditions alone and compared the effect of mood across the two flavor-type conditions. The interaction of flavor type and mood was significant $\left(F(1,199)=13.43, p<.001, \eta_{p}^{2}=.06\right)$. In same-flavor conditions, happy participants reported stronger preference for the first option than unhappy participants did $\left(M_{\text {happy }}=4.43\right.$ vs. $M_{\text {unhappy }}=6.40 ; t(199)=2.47, p<$ $.05, \eta_{p}^{2}=.03$; recall that smaller numbers indicate a preference toward the first option). A reverse pattern was observed under different-flavor conditions. Happy participants reported stronger preferences for the second option than unhappy participants did $(6.62$ vs. $4.42 ; t(199)=2.71$, $\left.p<.01, \eta_{p}^{2}=.04\right)$. These results are consistent with those from experiments 1 and 2 .

Second, to evaluate our assumption that the effects of mood on preference depended on the time at which the options were evaluated, we considered immediate-judgment and deferred-judgment conditions alone and analyzed preferences as a function of instructional condition (immediate vs. deferred), flavor type (same vs. different), and mood (positive vs. negative). It was expected that the instruction on the timing of evaluation would override the effect of flavor type in determining which option would be evaluated first and hence which option would be more subject to mood effect. This ANOVA yielded an interaction of mood and instructional conditions $\left(F(1,199)=12.14, p<.001, \eta_{p}^{2}=\right.$ .06 ) independent of flavor (as indicated by the nonsignificant three-way interaction, $F<1$ ). Planned comparisons further revealed that participants in immediate-judgment conditions preferred the first option more when they were happy $(M=$ 5.00 , averaged across the two flavor types) than when they were unhappy $(M=6.91$, averaged across the two flavor types; $\left.t(199)=3.03, p<.01, \eta_{p}^{2}=.04\right)$, whereas participants in deferred-judgment conditions preferred the second option more when they were happy $(M=6.03)$ than when they were unhappy $\left(M=4.81 ; t(199)=1.91, p=.06, \eta_{p}^{2}=.02\right)$.

Next, if the effect of mood under no-instruction, sameflavor conditions reflects a tendency to evaluate options at the time they are encountered, this effect may not differ from its effect in immediate-judgment conditions, but it should differ from its effect in deferred-judgment conditions. The no-instruction, same-flavor conditions were thus compared with immediate-judgment conditions and deferred-judgment conditions, respectively, through two interaction contrasts. The results revealed that the effect of mood in no-instruction, sameflavor conditions ( 4.43 vs. $6.40 ; M_{\text {diff }}=-1.97$ ) did not differ appreciably from that observed in immediate-judgment conditions ( 5.00 vs. $\left.6.91 ; M_{\text {diff }}=-1.91 ; F<1\right)$ but was opposite in direction to its effect in deferred-judgment conditions $(6.03$ vs. $\left.4.81 ; M_{\text {diff }}=1.22 ; F(1,199)=9.72, p<.01, \eta_{p}^{2}=.05\right)$.

Correspondingly, if the effect of mood under no-instruction, different-flavor conditions is attributable to a tendency to defer judgments until after all options have been presented, its effect should not differ appreciably from that observed in deferred-judgment conditions, but it should differ from its effect in immediate-judgment conditions. Interaction contrasts showed that the effect of mood in the no-instruction, different-flavor conditions (6.62 vs. 4.42 ; $\left.M_{\text {diff }}=2.20\right)$ did not differ from its effect in deferred-judgment conditions ( 6.03 vs. $\left.4.81 ; M_{\text {diff }}=1.22 ; F<1\right)$ but was opposite in direction to its effect in immediate-judgment conditions $\left(5.00\right.$ vs. $6.91 ; M_{\text {diff }}=-1.91 ; F(1,199)=16.00$, $\left.p<.01, \eta_{p}^{2}=.07\right)$. 
TABLE 5

INDEPENDENT EVALUATIONS AS A FUNCTION OF MOOD, PROCESS TYPE, AND FLAVOR TYPE CONDITIONS-EXPERIMENT 3

\begin{tabular}{|c|c|c|c|c|c|c|}
\hline 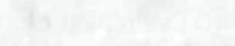 & \multicolumn{2}{|c|}{ No instruction } & \multicolumn{2}{|c|}{ Immediate judgment } & \multicolumn{2}{|c|}{ Deferred judgment } \\
\hline & $\begin{array}{c}\text { 1st } \\
\text { option }\end{array}$ & $\begin{array}{l}\text { 2nd } \\
\text { option }\end{array}$ & $\begin{array}{c}\text { 1st } \\
\text { option }\end{array}$ & $\begin{array}{l}\text { 2nd } \\
\text { option }\end{array}$ & $\begin{array}{c}\text { 1st } \\
\text { option }\end{array}$ & $\begin{array}{l}\text { 2nd } \\
\text { option }\end{array}$ \\
\hline \multicolumn{7}{|l|}{ Same flavor: } \\
\hline Positive mood & 7.76 & 6.57 & 7.48 & 6.93 & 6.63 & 6.69 \\
\hline Negative mood & 6.72 & 6.72 & 6.58 & 6.90 & 7.46 & 6.60 \\
\hline Difference & 1.04 & -.15 & .90 & .03 & -.83 & .09 \\
\hline \multicolumn{7}{|l|}{ Different flavor: } \\
\hline Positive mood & 6.38 & 6.89 & 7.90 & 6.75 & 7.46 & 6.52 \\
\hline Negative mood & 6.67 & 5.56 & 7.29 & 7.52 & 7.65 & 6.54 \\
\hline Difference & -.29 & 1.33 & .61 & -.77 & -.19 & -.02 \\
\hline
\end{tabular}

Independent Evaluations. Participants' evaluations of each option separately are shown in table 5. A mixed ANOVA using the two options' evaluations as a withinsubject factor (referred to as "option" hereafter) yielded a signification interaction of mood, flavor, process type, and option $\left(F(2,199)=4.54, p<.05, \eta_{p}^{2}=.04\right)$. Planned comparisons and interaction contrasts analogous to those performed on relative preference data provided support for our assumptions.

First, a mixed ANOVA based on evaluations under noinstruction conditions alone yielded an interaction of mood, flavor, and option $\left(F(1,199)=9.81, p<.01, \eta_{p}^{2}=.05\right)$, the nature of which confirms the results of the first two experiments. That is, in same-flavor conditions, mood had a greater effect on evaluations of the first option (7.76 vs. 6.72 , under positive vs. negative mood conditions, respectively; $\left.t(199)=2.04, p<.05, \eta_{p}^{2}=.02\right)$ than on evaluations of the second $(6.57$ vs. $6.72 ; t<1)$, whereas in different-flavor conditions, mood had a stronger influence on evaluations of the second option ( 6.89 vs. $5.56 ; t(199)=$ $\left.2.46, p<.05, \eta_{p}^{2}=.03\right)$ than the first $(6.38$ vs. $6.67 ; t<1)$.

Next, we ran a mixed ANOVA that focused on the two instructional conditions. It yielded an interaction of mood (positive vs. negative), instructions (immediate vs. deferred), and option $\left(F(1,199)=5.54, p<.05, \eta_{p}^{2}=.03\right)$ that was independent of flavor type $(F<1)$. Under immediate-judgment conditions, mood had an influence on evaluations of the first option ( $M=7.69$ vs. $M=6.94$, averaged across the two flavor types; $t(199)=1.86, p=.06, \eta_{p}^{2}=.02$ ) but not on evaluations of the second option (6.84 vs. 7.21; $t<1$ ). Contrary to our expectation, under deferred-judgment conditions, mood influenced neither the evaluation of the first option (7.05 vs. $7.55 ; t(199)=1.24, p=.21)$ nor the second option ( 6.61 vs. $6.57 ; t<1)$. We will discuss this further in the discussion.

Finally, we compared the findings from the no-instruction conditions to those from the instructional conditions. The effect of mood on evaluations of the two options under no-instruction, same-flavor conditions $\left(M_{\text {diff }}=1.04\right.$ and -.15 for the first and second options, respectively) did not differ from the effect under immediate-judgment conditions $\left(M_{\text {diff }}=.76\right.$ and -.37 , respectively; averaged over the two flavor types), as evidenced by an interaction of mood and option $\left(F(1,199)=5.50, p<.05, \eta_{p}^{2}=.03\right)$ that was independent of instructional conditions $(F<1)$. Corresponding contrasts under conditions where participants were expected to defer judgments are somewhat more equivocal. Mood had little effect on judgments of the first option in either the noinstruction, different-flavor or the deferred-judgment conditions, as expected ( $M_{\text {diff }}=-.29$ and -.51 , respectively). However, its effect on judgments of the second option was smaller in deferred-judgment conditions $\left(M_{\text {diff }}=.04\right)$ than in no-instruction, different-flavor conditions $\left(M_{\text {diff }}=1.33\right)$. This discrepancy from prediction is perplexing and is examined in more detail in the next experiment.

\section{Discussion}

Findings of this experiment were generally consistent with our expectations. However, the failure of mood to influence individual evaluations of the second option in deferred-judgment conditions is somewhat disconcerting. In fact, mood not only failed to influence evaluations of the second option but its effect on relative preference was only marginally significant $\left(M_{\text {happy }}=6.03\right.$ vs. $M_{\text {unhappy }}=4.81$; $t(199)=1.91, p=.06)$, despite the relatively large cell size after combining the two flavor types in these conditions. We suspected that this was due to an ineffective manipulation of judgment deferral. Specifically, suppose the instruction of "not to make any judgments until you have seen both options" was well received. The instruction might have prompted participants to think about avoiding making any immediate judgments when seeing the first option. This thought itself, however, might make the immediate-judgment goal even more accessible and, consequently, difficult to avoid (Higgins 1996; see also Wegner et al. [1987] for a similar finding). If this was the case, our participants might not have always, or uniformly, deferred their evaluations despite being instructed to do so. Although the measure on evaluation time showed that participants in deferred-judgment conditions had a relatively stronger disposition to defer their evaluations than those in immediate-judgment condi- 
TABLE 6

RELATIVE PREFERENCE AND INDEPENDENT EVALUATIONS AS A FUNCTION OF MOOD AND PROCESS INSTRUCTION-EXPERIMENT 4

\begin{tabular}{|c|c|c|c|}
\hline & Relative preference & $\begin{array}{l}\text { Evaluation of } \\
\text { the } 1 \text { st option }\end{array}$ & $\begin{array}{l}\text { Evaluation of } \\
\text { the } 2 \text { nd option }\end{array}$ \\
\hline \multicolumn{4}{|l|}{ No instruction, different flavor: } \\
\hline Positive mood $(n=18)$ & 5.94 & 6.06 & 6.33 \\
\hline Negative mood $(n=18)$ & 4.50 & 6.28 & 4.83 \\
\hline \multicolumn{4}{|c|}{ Deferred judgment, same flavor: } \\
\hline Positive mood $(n=17)$ & 6.24 & 5.88 & 6.29 \\
\hline Negative mood $(n=16)$ & 4.75 & 6.69 & 4.75 \\
\hline \multicolumn{4}{|c|}{ Deferred judgment, different flavor: } \\
\hline Positive mood $(n=17)$ & 6.00 & 6.06 & 6.47 \\
\hline Negative mood $(n=17)$ & 4.71 & 6.24 & 5.12 \\
\hline
\end{tabular}

tions, this might reflect a demand effect in the deferredjudgment conditions because an explicit instruction of judgment deferral was given to participants in these conditions. To this extent, the deferred-judgment conditions may not be an unambiguous standard for evaluating the results in the no-instruction, different-flavor conditions. The next experiment attempted to eliminate this problem.

\section{EXPERIMENT 4}

\section{Method}

This experiment was restricted to the three conditions in which participants were expected to judge the final option first (i.e., the "no-instruction, different-flavor," "deferredjudgment, same-flavor," and "deferred-judgment, differentflavor" conditions). However, the procedure used in the two deferred-judgment conditions was modified. Instead of presenting the materials as part of a comparison task, the experimenter told participants that we were concerned with how individuals remember pictorial information and asked them to try to remember the pictures conveyed in the materials as well as they could. After presenting the two options sequentially, as in experiment 3 , participants were unexpectedly asked to evaluate the second option, followed by the first option, and then to indicate their relative preference. This procedure provided a more unambiguous baseline for comparing with no-instruction, different-flavor conditions. Moreover, as judgment deferral was not enforced by using any explicit instructions, we could avoid the potential problems associated with the use of such an instruction as in experiment 3 .

One hundred and three participants were randomly assigned to each of the six conditions of a 3 (process type: no instruction, different flavor vs. deferred judgment, same flavor vs. deferred judgment, different flavor) $\times 2$ (induced mood: positive vs. negative) factorial design.

\section{Results}

Mood Manipulation Check. The mood induction procedure was successful. Participants reported feeling happier after describing their experience in the positive mood condition than in the negative mood condition (4.44 vs. -2.31 ; $\left.F(1,97)=86.91, p<.001, \eta_{p}^{2}=.47\right)$.

Timing of the First Evaluation. The time at which participants reported making their first evaluation was computed as in experiment 3. Participants in instructional (deferred-judgment) conditions showed a similar tendency to do so regardless of whether the options had the same flavor $(M=3.29)$ or different flavors $(M=3.31 ; t<1)$. Moreover, this tendency did not differ from that observed under no-instruction conditions $(M=3.56 ; t<1)$. Neither the main effect of process type nor the interaction between mood and process type was significant ( $F<1$ in both cases).

\section{Relative Preference and Independent Evaluations.}

The relative preference data, as shown in table 6 , replicated the findings of experiment 3 . A 3 (process type) $\times 2$ (mood) ANOVA yielded a main effect of $\operatorname{mood}(F(1,97)=11.31$, $\left.p<.01, \eta_{p}^{2}=.10\right)$ independent of process type $(F<1)$. A planned comparison suggested that participants under the noinstruction conditions had a stronger preference for the second option when they were in a positive $\operatorname{mood}(M=5.94)$ than when they were in a negative $\operatorname{mood}(M=4.50 ; t(97)=$ $\left.2.04, p<.05, \eta_{p}^{2}=.04\right)$. Similarly, participants under the two instructional conditions had a stronger preference for the second option when they were in a positive $\operatorname{mood}(M=6.12$, averaged across the two flavor types) than when they were in a negative mood $(M=4.73$, averaged across the two flavor types; $t(97)=2.68, p<.01, \eta_{p}^{2}=.07$ ), and the difference did not differ across the two flavor type conditions $(F<1)$. An additional interaction contrast showed that effects of mood under the instructional conditions did not differ from those observed under the no-instruction conditions $(F<1)$.

The evaluation data were also consistent with expectations. A 2 (the two options' evaluations) $\times 2$ (mood) $\times 3$ (process type) mixed ANOVA yielded a significant inter- 
action between mood and option $(F(1,97)=11.36, p<$ $\left..01, \eta_{p}^{2}=.11\right)$ that did not depend on process type $(F<1)$. As expected, happy and unhappy participants in the noinstruction conditions did not differ in terms of their evaluations of the first option $(6.06$ vs. $6.28 ; t<1)$. This was also the case for the two instructional conditions (5.97 vs. $6.46 ; t(97)=1.21, p=.25$; means were average of the two flavor types, as the evaluations did not differ appreciably as a function of flavor type, $F<1$ ). Of central interest is the evaluation of the second option. Participants in noinstruction conditions evaluated the second option more favorably when they were happy than when they were unhappy ( 6.33 vs. $\left.4.83 ; t(97)=2.19, p<.05, \eta_{p}^{2}=.05\right)$. A similar pattern was observed under instructional conditions ( 6.38 vs. 4.93 , averaged across the two flavor types; $\left.t(97)=2.88, p<.01, \eta_{p}^{2}=.08\right)$, which did not differ across the two instructional conditions $(F<1)$. Additional interaction contrast suggested that effects of mood under noinstruction conditions did not differ from those observed under the instructional conditions $(F<1)$.

\section{Discussion}

Findings of this experiment indicated that participants in deferred-judgment conditions had held off their judgments until they had seen the second option and attributed their feelings to the second option rather than the first presented option. This also appeared to be the way that participants in no-instruction conditions made their comparison. Thus, in combination with the evidence obtained in experiment 3 , these results provide good evidence that the influence of mood on comparison depends on which alternative is the one being evaluated first. That is, when participants evaluated the options starting with the first, they preferred the first option more when they were in a positive mood than when they were in a negative mood, whereas when participants evaluated the options starting with the last, they preferred the last option more when they were in a positive mood than when they were in a negative mood.

It would nevertheless be inappropriate to conclude that consumers always defer their evaluations whenever the choice scenario resembles the one in the different-flavor condition. Findings of experiment 3 indicate that people may not uniformly hold off evaluation even when they are explicitly instructed to do so. Having said that, however, we have to emphasize that the relationship between evaluation time and choice set characteristics is not the point of theoretical interest in itself in the present research. Therefore, the findings based on the no-instruction, different-flavor and the deferred-judgment conditions alone are not definitive. Rather, the findings in combination, coupled with findings from the no-instruction, same-flavor and the immediatejudgment conditions provide evidence for the proposition that the influence of mood on comparison depends on which alternative is the one being evaluated first.

\section{GENERAL DISCUSSION}

A substantial amount of research has been devoted to understanding the influence of affect on consumer judgment (see Pham [2004] for a review). However, it is surprising that little research has been done to examine how affect influences comparisons and choices. Our research fills this gap by demonstrating a systematic influence of mood on choice, which contrasts with the general assumption that mood is unlikely to influence choice. In the first experiment, we showed that when participants were disposed to evaluate each product at the time it was presented, mood influenced the evaluation of the first presented option rather than those of the subsequently presented options. Consequently, mood influenced choice of the first presented option. However, in the second experiment, when participants were disposed to withhold their evaluations until all of the options had been presented, mood appeared to influence the evaluation and the choice of the last presented option. Then, we provided evidence that the different effect of mood across these experiments was due to the different timing at which the first evaluation was constructed in the course of comparison. Altogether, these findings suggest that the influence of mood on comparison depends on which alternative in a choice set is the one being evaluated first.

Several aspects of our research are worthy of further discussion. First, in the present research, we consider comparisons that are made based on global evaluations of choice alternatives. This is obviously not the only way to make comparisons. In the consumer choice literature, researchers distinguish between comparisons that are conducted in an attribute-by-attribute manner and those that are based on global evaluations of choice alternatives (see Bettman, Johnson, and Payne [1991] for a review). The strategy we consider in the present research is more consistent with the latter type of comparison. We, of course, expect the influence of mood on comparison to be different depending on how it is actually conducted. For this reason, we define a domain for the present research and control for the comparison strategy that participants employed in our experiments. The stimuli we employed were therefore designed to facilitate the formation of global evaluations and the use of these evaluations as a basis of comparison. For example, we presented pictures showing alternatives' appearance, which is likely to stimulate the formation of global evaluations. Also, we provided very limited descriptive information so that participants were unlikely to engage in attribute-based comparison. A worthwhile avenue for further research would be to extend the domain for investigation and examine the influence of mood in attribute-based comparison. For example, because attribute-based comparison requires the comparison of specific attributes associated with each alternative, it has a certain level of cognitive requirement (Mantel and Kardes 1999). Thus, if affect influences a decision-maker's motivation to engage in cognitive activities (Schwarz and Clore 1996), or if it influences the ability to process information (Eysenck 1982), it may potentially influence the tendency to conduct an attribute-by-attribute comparison. Moreover, 
if the alternatives' attributes differ in valence, feelings may influence how the attributes are attended to, encoded, and weighted (Adaval 2001; Forgas and Bower 1987) in the course of comparison. In any case, while we expect the existing affect theories in the independent judgment domain to be generalizable to the comparative judgment domain, we also expect to see some distinct influences of affect on comparative judgment that could not be directly extended from the previous research. Therefore, this would be an interesting avenue for future research.

Second, the present research is restricted to conditions in which people interpret their feelings as an indication of how much they like a target and use these feelings as a basis for evaluating the target (i.e., the "affect-as-information" conceptualization; Schwarz 1990). Our assumption that mood influences the evaluation of an alternative at the precise time the evaluation is constructed (Srull 1987) follows directly from the affect-as-information conceptualization. This assumption, however, is not entirely consistent with other theories that suggest that mood can activate mood-consistent thoughts and, therefore, influence the manner in which people process information and make judgments (e.g., Bower 1981; for a review, see Forgas [1995]). Although this latter theory did not seem to explain the findings in the present research, it would be inappropriate to conclude that mood would not influence comparison through the activation of feeling-consistent thoughts. As discussed earlier, it is intuitively likely that the influence of mood on comparison through the activation of feeling-consistent cognitions will be more prominent in attribute-based comparisons. This would be another worthwhile avenue for further investigation.

Third, our finding shows that when alternatives under consideration are characterized by a descriptive feature, consumers are likely to defer their evaluations until after they have seen the last alternative. Nevertheless, this finding should not be overgeneralized, as it may only be true when consumers consider a limited number of options. Presumably, if consumers want to wait until they have seen the last option to make a judgment, they have to remember the descriptive features of each encountered option. It is, however, impossible to memorize all the alternatives' descriptive features if they have a large choice set to consider. Future empirical research is needed to extend the existing findings to this type of choice situation.

In a related vein, our finding shows that choice set configuration may be an important determinant of comparison strategy. We find that when a choice set is configured in such a way that the alternatives differ in only external appearance, consumers tend to evaluate the first presented option immediately. However, when alternatives differ in descriptive features that are more central to judgment, consumers tend to defer their evaluations to a later time. We attributed this difference to the higher perceived diagnosticity of global, appearance-based evaluations as a basis for choice in the former case than in the latter case. However, we have not provided an empirical test of this underlying assumption. Future research is needed to provide a more systematic examination of the underlying mechanism, as well as the effect of other situational influences on comparison strategy.

Aside from the above issues, we would like to point out that although we have obtained support across experiments for our theoretical framework, the effect sizes are quite modest. The importance of this research, however, should not be judged by the magnitude of effect sizes. Like most consumer behavior research, our research selected the levels of the factors systematically based on theoretical considerations, which in the first place makes the effect-size estimates less informative for judging theoretical importance (see Fern and Monroe [1996] for a discussion of the difference between these models and the implications to effect sizes). Moreover, our findings provide evidence contrasting the viewpoint that existing theoretical frameworks on subjective experience effects (e.g., mood, metacognitive experience) have limited applicability in the choice domain. Thus, the presence of an effect on choice, regardless of its size, explicates an important theoretical point.

\section{REFERENCES}

Adaval, Rashmi (2001), "Sometimes It Just Feels Right: The Differential Weighting of Affect-Consistent and Affect-Inconsistent Product Information," Journal of Consumer Research, 28 (June), 1-17.

Agresti, Alan (1996), An Introduction to Categorical Data Analysis, New York: Wiley \& Sons.

Bettman, James R., Eric J. Johnson, and John Payne (1991), “Consumer Decision Making," in Handbook of Consumer Behavior, ed. Thomas S. Robertson and Harold H. Kassarjian, Englewood Cliffs, NJ: Prentice-Hall.

Bower, Gordon H. (1981), "Mood and Memory," American Psychologist, 36, 129-48.

Eysenck, M. W. (1982), Attention and Arousal, Cognition and Performance, New York: Springer.

Gorn, Gerald J., Marvin E. Goldberg, and Kunal Basu (1993), "Mood, Awareness, and Product Evaluation," Journal of Consumer Psychology, 2 (3), 237-56.

Fern, Edward F. and Kent B. Monroe (1996), "Effect Size Estimates: Issues and Problems in Interpretation," Journal of Consumer Research, 23 (September), 89-105.

Forgas, Joseph P. (1995), "Mood and Judgment: The Affect Infusion Model (AIM)," Psychological Bulletin, 117, 39-66.

Forgas, Joseph P. and Gordon H. Bower (1987), "Mood Effects on Person-Perception Judgments," Journal of Personality and Social Psychology, 53 (July), 53-60.

Higgins, E. Tory (1996), "Knowledge Activation: Accessibility, Applicability, and Salience," in Social Psychology: Handbook of Basic Principles, ed. E. Tory Higgins and Arie W. Kruglanski, New York: Guilford, 133-68.

Houston, David A., Steven J. Sherman, and Sara M. Baker (1989), "The Influence of Unique Features and Direction of Comparison on Preferences, "Journal of Experimental Social Psychology, 25 (March), 121-41.

Huber, Joel (2004), "A Comment on Metacognitive Experiences and Consumer Choice" (invited comment), Journal of Consumer Psychology, 14 (4), 356-59. 
Keppel, Geoffrey (1991), Design and Analysis: A Researcher's Handbook, 3rd ed., Englewood Cliffs, NJ: Prentice-Hall.

Mantel, Susan P. and Frank R. Kardes (1999), "The Role of Direction of Comparison, Attribute-Based Processing, and Attitude-Based Processing in Consumer Preference," Journal of Consumer Research, 25 (March), 335-52.

Novemsky, Nathan, Ravi Dhar, Norbert Schwarz, and Itamar Simonson (2007), "Preference Fluency in Consumer Choice," Journal of Marketing Research, 44 (August), 347-56.

Pham, Michel T. (1998), "Representativeness, Relevance, and the Use of Feelings in Decision Making," Journal of Consumer Research, 25 (September), 144-59.

- (2004), "The Logic of Feeling," Journal of Consumer Psychology, 14 (4), 360-69.

Pham, Michel T., Joel B. Cohen, John W. Pracejus, and G. David Hughes (2001), "Affect Monitoring and the Primacy of Feelings in Judgment," Journal of Consumer Research, 28 (September), 167-88.

Pham, Michel Tuan and Tamar Avnet (2004), "Ideals and Oughts and the Reliance on Affect versus Substance in Persuasion," Journal of Consumer Research, 30 (March), 503-18.

Schwarz, Norbert (1990), "Feelings as Information: Informational and Motivational Functions of Affective States," in Handbook of Motivation and Cognition, Vol. 2, ed. Richard. M. Sorrentino and E. Tory Higgins, New York: Guilford, 521-61. (2001), "Feelings as Information: Implications for Affective Influences on Information Processing," in Theories of Mood and Cognition: A User's Guidebook, ed. Leonard L. Martin and Gerald L. Clore, Mahwah, NJ: Erlbaum, 159-76.

Schwarz, Norbert and Gerald L. Clore (1983), "Mood, Misattribution, and Judgments of Well-Being: Informative and Directive Functions of Affective States," Journal of Personality and Social Psychology, 45 (September), 513-23.

(1988), "How Do I Feel about It? The Informative Functions of Affective States," in Affect, Cognition, and Social Behavior, ed. Klaus Fiedler and Joseph Forgas, Toronto: Hegrefe International, 44-62.

(1996), "Feelings and Phenomenal Experiences," in Social
Psychology: Handbook of Basic Principles, ed. E. Tory Higgins and Arie W. Kruglanski, New York: Guilford, 433-65. Schwarz, Norbert, Wolfgang Servay, and Martin Kumpf (1985), "Attribution of Arousal as a Mediator of the Effectiveness of Fear-Arousing Communications," Journal of Applied Social Psychology, 15, 74-78.

Shiv, Baba and Alexander Fedorikhin (1999), "Heart and Mind in Conflict: The Interplay of Affect and Cognition in Consumer Decision Making," Journal of Consumer Research, 26 (December), 278-92.

(2002), "Spontaneous versus Controlled Influences of Stimulus-Based Affect on Choice Behavior," Organizational Behavior and Human Decision Processes, 87 (March), 342-70.

Siemer, Matthias and Rainer Reisenzein (1998), "Effects of Mood on Evaluative Judgments: Influence of Reduced Processing Capacity and Mood Salience," Cognition and Emotion, 12 (November), 783-805.

Srull, Thomas K. (1987), "Memory, Mood, and Consumer Judgment," Advances in Consumer Research, 14, 404-7.

Strack, Fritz (1992), "The Different Routes to Social Judgments: Experiential versus Informational Strategies," in Construction of Social Judgments, ed. Leonard L. Martin and Abraham Tesser, Hillsdale, NJ: Erlbaum, 249-75.

Wang, Jing and Robert S. Wyer (2002), "Comparative Judgment Processes: The Effects of Task Objectives and Time Delay on Product Evaluations," Journal of Consumer Psychology, 12 (4), 327-40.

Wegner, Daniel M., David J. Schneider, Samuel R. Carter III, and Teri L. White (1987), "Paradoxical Effects of Thought Suppression," Journal of Personality and Social Psychology, 53 (1), 5-13.

Yeung, Catherine W. M. and Robert S. Wyer (2004), "Affect, Appraisal, and Consumer Judgment," Journal of Consumer Research, 31 (September), 412-24.

(2005), "Does Loving a Brand Mean Loving Its Products? The Role of Brand-Elicited Affect in Brand Extension Evaluations," Journal of Marketing Research, 42 (November), 495-506. 
Copyright of Journal of Consumer Research is the property of Journal of Consumer Research. Inc. and its content may not be copied or emailed to multiple sites or posted to a listserv without the copyright holder's express written permission. However, users may print, download, or email articles for individual use. 